\title{
Comparing the steric height in the Northern Atlantic with satellite altimetry
}

\author{
V. O. Ivchenko ${ }^{1}$, S. D. Danilov ${ }^{1}$, D. V. Sidorenko ${ }^{1}$, J. Schröter ${ }^{1}$, M. Wenzel ${ }^{1}$, and D. L. Aleynik ${ }^{2}$ \\ ${ }^{1}$ Alfred Wegener Institute for Polar and Marine Research, Bremerhaven, Germany \\ ${ }^{2}$ The University of Plymouth, Plymouth, UK \\ Received: 23 April 2007 - Published in Ocean Sci. Discuss.: 16 May 2007 \\ Revised: 12 October 2007 - Accepted: 15 November 2007 - Published: 28 November 2007
}

\begin{abstract}
Anomalies of dynamic height derived from an analysis of Argo profiling buoys data are analysed to assess the relative roles of contributions from temperature and salinity over the North Atlantic for the period of 1999-2004. They are compared with dynamic topography anomalies based on TOPEX/Poseidon and Jason altimetry. It is shown that the halosteric contribution to the anomalies of dynamic height is comparable in magnitude to the thermosteric one over the period analyzed. Taking both salinity and temperature into account improves the agreement between zonally averaged trends in the satellite dynamic topography and dynamic height increasing the correlation between them to 0.73 from 0.63 when only temperature variability is taken into account. The implication of this result is that the salinity contribution cannot be neglected in the North Atlantic and that one cannot rely on estimating the thermosteric part by anomalies in the sea surface dynamic topography derived from the satellite altimetry.
\end{abstract}

\section{Introduction}

Whether the ocean is warming or cooling is an important question both in physical oceanography and for monitoring climate change. Temperature anomalies in the ocean lead to changes in the steric height and thus contribute to sea surface height (SSH) anomalies which can also be observed from satellites. This suggests using satellite altimetry to improve estimates of heat content tendencies (Willis et al., 2004) still suffering from sparsity of the in-situ data.

However, the variability of the SSH can be caused by different oceanic processes. In addition to steric height changes it is affected by redistribution of the water masses in the ocean or barotropic component of sea-surface height (Willis

Correspondence to: V. O. Ivchenko

(voi@noc.soton.ac.uk) et al., 2003, 2004), or direct changes in the total ocean mass (sea level rise). The steric height variability itself depends not only on temperature but also on salinity anomalies. Thus the temperature signal in the SSH anomalies is masked by other influences.

Many authors suggest that the temperature anomalies in the upper few hundred meters is the leading factor determining both steric height and SSH anomalies and disregard the influence of the salinity variation. Such analyses are made either for the global ocean (Willis et al., 2003) or for its particular regions (White and Tai, 1995). Note, that even if the thermosteric effect dominates over the halosteric one in the globally averaged steric height, this is not necessarily true locally. Antonov et al. (2002) point out that the salinity effects are crucially important in some regions, including the subpolar North Atlantic. However, the haline effects play a minor role for the spatially averaged steric height between $50^{\circ} \mathrm{S}$ and $65^{\circ} \mathrm{N}$ for the 1957-1994 period. Sato et al. (2000) demonstrate importance of salinity for the heat storage estimation from TOPEX/Poseidon in the in situ data for three points at low latitudes in the Pacific and Atlantic. Gilson et al. (1998) demonstrate that including salinity measurements significantly reduces errors in specific volume and steric height in the North Pacific. Maes (1998) discusses different possible mechanisms in tropics, subtropics and the Southern Ocean by which the salinity variations may generate a signal in sea level.

Summing up, the following problems need to be resolved:

- What is the relative importance of variability of salinity and temperature in their contribution to the steric height?

- Can other mechanisms, like barotropic motions (seen in bottom pressure anomalies) lead to strong deviations of the steric height variability from sea level variability? On what time scales does it happen? 


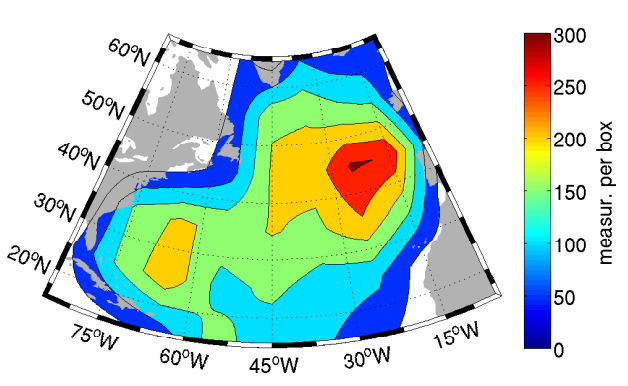

Fig. 1. The number of the temperature profiling data at the surface of the North Atlantic for the $10^{\circ}$ boxes for the 1999-2005.

- In which regions can one expect increased halosteric contributions?

The availability of the Argo floats data suggests a unique possibility to address these questions which is the main goal of this paper. Our analysis below is limited to the North Atlantic basin and mainly concentrated on the first problem. We should add the caveat that period of five years is insufficient for an accurate computation of the ocean trends.

We describe the data used and their preparation in Sect. 2. Sections 3-5 present our main results, discussion and conclusion, respectively.

\section{Data and method}

Temperature and salinity profiles from the Argo project were used for calculations of steric height. The analysis is limited to the North Atlantic basin between $10^{\circ} \mathrm{N}$ and $70^{\circ} \mathrm{N}$ and to the period between January 1999 to December 2004. The data were obtained from the official Argo web-site. They comprise 31949 temperature profiles and 19108 salinity profiles. Quality control flags provided by the originators were used to eliminate inappropriate profiles, which led to some reduction of the data set. The data set was further reduced by eliminating profiles that differ by more than 4 standard deviations from the monthly averaged values in 10 by 10 degree boxes. In the February 2007 the Argo users were informed that profiles from SOLO floats with FSI CTD (Argo Program WHOI) may have incorrect pressure values. For this reason all observations by this type of instrument were removed from our data set. The number of profiles decreases to 22529 and 16088 for the temperature and salinity data, respectively.

The mean coverage of the Northern Atlantic with floats is shown in Fig. 1a. It is not uniform with a maximum over the central North Atlantic and minima along the coast. The coastal regions correspond to about 10 profiles in a 10 by 10 degree box per month of observations. It is worth mentioning that there is considerable improvement in coverage of the North Atlantic from 1999 to 2004 almost everywhere, except for the western part of the zonal belt between $20^{\circ} \mathrm{N}$ and $30^{\circ} \mathrm{N}$ and the north-eastern part of the basin. The amount of the data depends strongly on the depth. It drops by about $25 \%$ at $500 \mathrm{~m}$ depth and decreases further down.

The temperature and salinity differences between the observed (Argo) values and monthly WOA2001 climatology (Stephens et al., 2002) (interpolated to the point of observation) were calculated. The anomalies of temperature and salinity fields were calculated with an objective analysis scheme based on Gandin (1963) and Bretherton et al. (1976) which is similar to that of Lavender et al. (2005) and Ivchenko et al. (2006). It gives a linear unbiased estimate which is optimal in the least squares sense. The covariance of the data is assumed to be Gaussian with the correlation length $L=350 \mathrm{~km}$ everywhere within the domain. This value is more than twice the value used by Lavender et al. (2005), but is justified by the data distribution within the domain used here.

The altimetry data set used for comparison is based on the TOPEX/Poseidon/Jason missions and was provided to us by S. Esselborn (GFZ Potsdam). It covers a period from 1993 to May 2005. Both Argo and altimetry monthly mean anomalies were interpolated to 1 by 1 degree grid.

The steric height is defined as an integral between the surface and some depth $D$ (or pressure) of the specific volume anomaly $\delta$ computed with respect to a temperature of $0^{\circ} \mathrm{C}$ and a salinity of $35 \mathrm{psu}$ (Gill, 1982). A depth of $1000 \mathrm{~m}$ was used for the most of the presented results (see Sect. 4). The monthly anomalies of steric height (ASH) were computed with respect to their mean annual cycle over January 1999December 2004 while the mean annual cycle over a longer period of time (1993-December 2004) was used to compute anomalies of satellite altimetry (ASA). Due to difference in averaging periods there is offset of the ASA with respect to ASH. It is computed as the difference between mean values of the ASH and ASA over the last six years and compensated for the comparison of the anomalies.

The error estimates for trends $\sigma_{\alpha}$ are computed as $\sigma_{\alpha}=$ $\frac{\sigma_{f l d}}{\sigma_{[1 \ldots n]}} \cdot \frac{1-r^{2}}{(n-1)^{1 / 2}}$, where $\sigma_{f l d}$ is the standard deviation of a field (sea surface satellite altimetry or steric height) for $n$ years, $\sigma_{[1 \ldots n]}$ is the square root of the variance of the set of years from 1 to $n$, and $r$ is the correlation coefficient between the regression and the variability of the field.

\section{Results}

\subsection{Steric height vs. altimetry}

A comparison between the ASA and ASH for the anomalies integrated over the entire basin is shown in Fig. 2. As follows from panels A and B of Fig. 2, the seasonal cycles of the steric height and altimetry are very similar with the amplitude of the altimetry being higher by about $1.2 \mathrm{~cm}$. Since the depth of $1000 \mathrm{~m}$ well exceeds the depth of the seasonal 

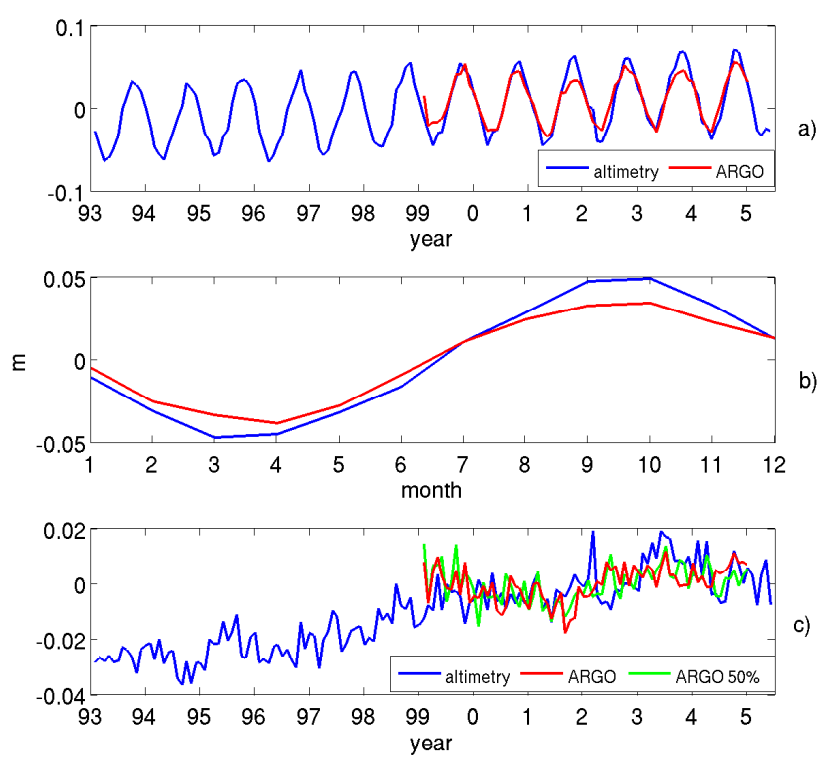

Fig. 2. The ASH (red) and ASA (blue) anomalies averaged over the Northern Atlantic. (a) - full anomalies; (b) - the seasonal cycle of the ASH and ASA; the averaging time is between 1999 and 2005 and between 1993 and 2005 for the ASH and ASA, respectively; (c) - The anomalies after removing the seasonal cycle. The green curve shows ASH for the experiment with the half of the data excluded. The ASA curve is shifted down by the difference between the mean over last six years and the total mean. The units are in $\mathrm{m}$.

thermocline this difference should be attributed mostly to non-steric contributions into the ASA.

Removing the seasonal cycle of both data sets leaves anomalies of smaller amplitude which are considered further. Panel C of Fig. 2 shows the basin averaged ASH (red) and ASA (blue) for both data sets. They are generally consistent and show variability of similar amplitude within individual years. The trend for the ASA is $2.58 \mathrm{~mm} /$ year and larger than the trend of the ASH, which is $1.44 \mathrm{~mm} /$ year with standard errors of $0.38 \mathrm{~mm} /$ year and $0.37 \mathrm{~mm} /$ year for the ASA and ASH, respectively. The trends are computed over last six years.

Annually averaged fields of the ASH and ASA were computed to exclude the influence of the seasonal cycle and variability on short time scales. The differences in horizontal distributions between two consecutive years (not reproduced here) have much in common for ASH and ASA in spite of their inherently different patchiness.

In order to simultaneously characterize the entire period of observation we computed linear regression for five annual mean values at every grid point for ASH and ASA (i.e. 20002004; 1999 was excluded because salinity measurements are less dense). Figure 3 depicts the pattern of trend found for the satellite data (Fig. 3a) and steric height (Fig. 3b). The values of the trend of the surface height and the steric height are larger than the values of the errors shown in Fig. 3c, d.
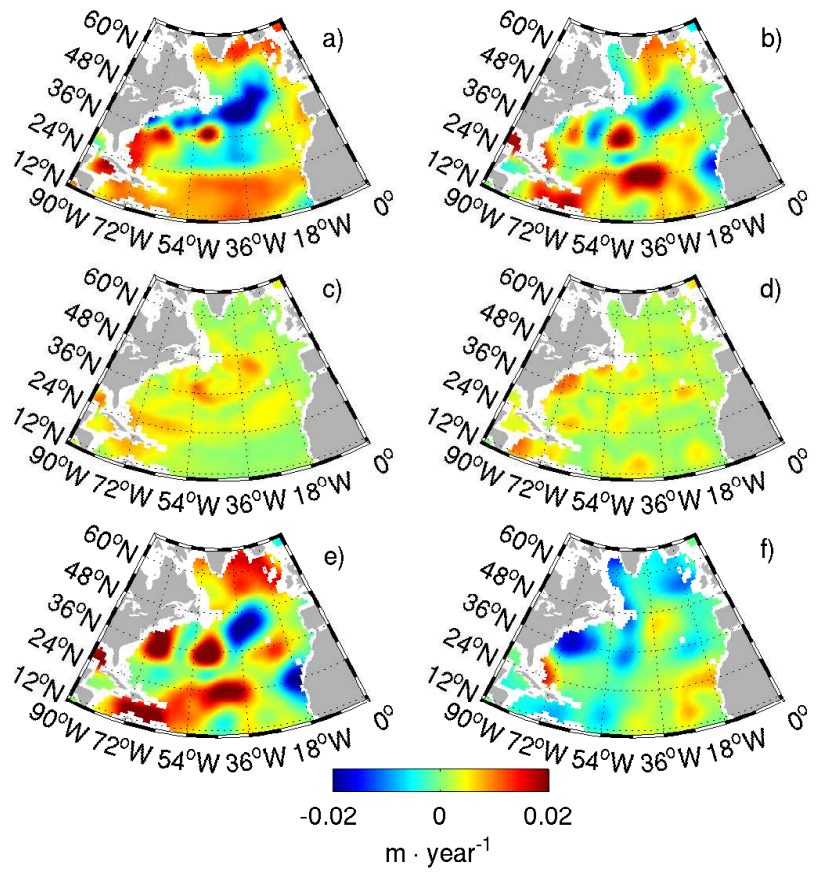

Fig. 3. The trends of the altimetry and steric height anomalies for the period 2000-2004. Panels (a), (b), (e) and (f) correspond to trends in satellite altimetry, full steric height and the thermosteric and halosteric contributions, respectively. Panels (c) and (d) present trend uncertainties for ASA and ASH respectively.

Once again, there is a reasonable similarity between the trend patterns. Predominantly positive trends occur in the southern, northern and mid western domains of the basin in both fields. Mostly negative values are in the mid North Atlantic and subpolar gyre in both fields. However, contrary to the negative trend of the ASH in the south-east corner of the basin the trend in the ASA is positive here with exception of small area in the vicinity of Africa. A similar difference is found for the Labrador Sea where we see a negative trend in the steric height but a positive trend in the satellite altimetry. A possible reason for such a difference in behavior can be a bias due to insufficient amount of the Argo data in these regions (see Fig. 1).

\subsection{Roles of temperature and salinity anomalies}

Computing the thermosteric and halosteric ASH allows for the study of the roles of these anomalies separately. The "temperature only" (or "salinity only") experiment uses real temperature (salinity) data, but climatological salinity (temperature). Because of nonlinearity of the equation of state the "temperature only" and "salinity only" anomalies do not make up the full response when summed. Yet the separation is convenient to assess the magnitudes of contributions due to temperature and salinity. The anomaly patterns computed for separate years reveal that salinity and 


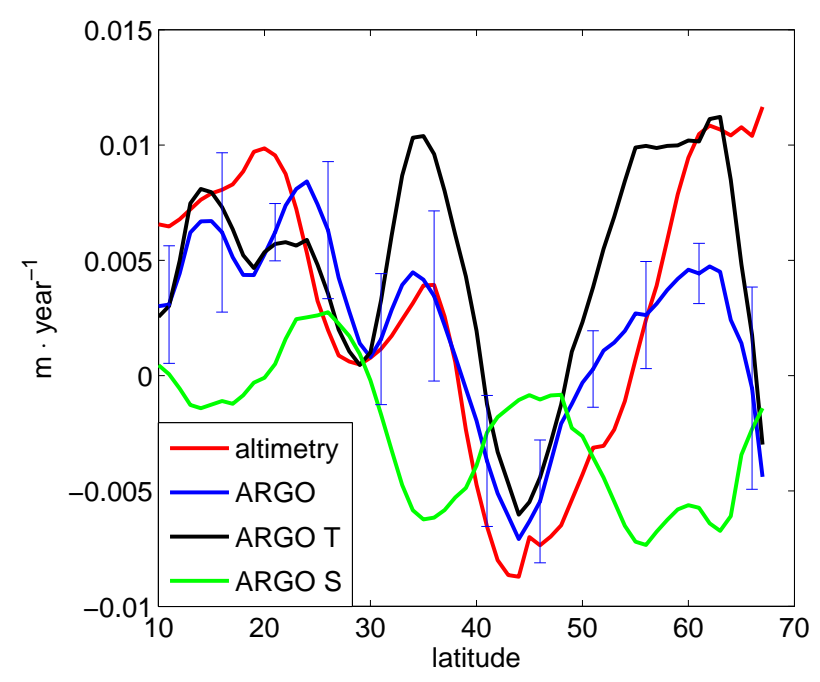

Fig. 4. Zonally averaged trends between 2000 and 2004 for the satellite altimetry (red), full steric height (blue), and thermosteric (black; salinity is climatological)) and halosteric (green; temperature is climatological)) heights. The vertical bars represent uncertainties.

temperature contributions into ASH are of similar magnitude (several centimeters) although the pattern of thermal contribution in the ASH is closer to ASA. In many places, the salinity anomalies act against the temperature anomalies. This fact is best seen in patterns of trend due to thermal expansion and saline contraction shown in Fig. 3e and $f$ respectively.

The main contribution to the profound positive signal in the northern and southern domains comes from temperature anomalies. Similarly, a predominantly negative trend in the ASH above the center of the North Atlantic Ridge comes from the temperature. However, the importance of the contribution of salinity anomaly is clearly seen over the eastern domains and close to the U.S. coast starting at $30^{\circ} \mathrm{N}$. A strong positive trend of the ASH near the African coast (which is not observed in the satellite altimetry trend) is partly compensated by the strong negative ASH coming from the halosteric part.

In order to estimate a typical value of local trends we computed their rms values. The rms value of the trend of the ASA is higher than that of the steric height $(8.5$ and $7.7 \mathrm{~mm}$ /year, respectively). The rms value of the steric height lies between the corresponding values of the "temperature only" and "salinity only" experiments which are 9.6 and $5.6 \mathrm{~mm} / \mathrm{year}$, respectively. Thus the impact of salinity anomalies on the steric height is to partly compensate the temperature influence.

There is a strong similarity between the zonally averaged trends of ASH and ASA as demonstrated by Fig. 4. The correlation between the corresponding curves is relatively high and reaches 0.73 . The correlation between thermosteric anomalies and ASA drops down to 0.63 while it becomes negative $(-0.56)$ between the thermosteric and halosteric contributions. Disregarding the influence of salinity leads to an overestimation of the steric height and reduces the correlation between ASA and ASH.

One expects that on interannual time scale an essential part of variability in temperature and salinity within the main thermocline is induced by motion of isopycnals as a response to wind anomalies. This implies negative spatial correlation between thermal and saline contributions to anomalies of density on geopotential surfaces. Similarly, adiabatic displacements of water parcels along isopycnals also lead to anticorrelated changes in temperature and salinity. That is why our result is not unexpected.

To illustrate this issue we computed yearly averaged anomalies of the depth of neutral density surface $\gamma=27.5$ (ADNDS). The differences in ADNDS between consecutive years are mainly due to the temperature variability for the first several years (2000-1999 and 2001-2000). This can be partly a consequence of inadequate coverage of salinity data for the first two years. In the following years (2002-2001 and 2003-2002), the contribution from the "salinity only" is even stronger than the contribution from the "temperature only".

The change of depth of the neutral surface $\gamma=27.5$ between 2004 and 2003 in Fig. 5 demonstrates the importance of both temperature and salinity contributions in forming this change. A large negative displacement of the neutral surface in the eastern southern and middle parts is apparently associated with salinity anomalies. Over other places like the western middle part and the northern part the positive values are mainly related to the temperature field. The rms displacement due to both temperature and salinity anomalies is $16.9 \mathrm{~m}$. Thermally induced $\mathrm{rms}$ displacement is larger $(20.2 \mathrm{~m})$, and the rms displacement due to "salinity only" is $19.6 \mathrm{~m}$.

\section{Discussion}

An important question to be answered is whether the Argo data coverage is good enough to calculate the steric height and heat content. The short answer is "yes" for the temperature dependent fields (heat content, thermosteric contribution to ASH). The salinity coverage is patchy over the first three years, but becomes reliable afterwards. Apparently, the fine structure of anomalies in temperature, salinity and steric height (as seen in their horizontal distributions) is affected by objective interpolation. Given the techniques used in the present analysis, denser sampling is required to provide reliable comparison with altimetric anomalies over the entire North Atlantic. However, zonal and basin averages show consistent behavior. We checked, for example, that removing $50 \%$ of data does not dramatically change the results of the domain averaged heat content or steric height. Indeed, anomaly of SH for the total set of the data with 50\% of Argo excluded at random demonstrates a distribution very 

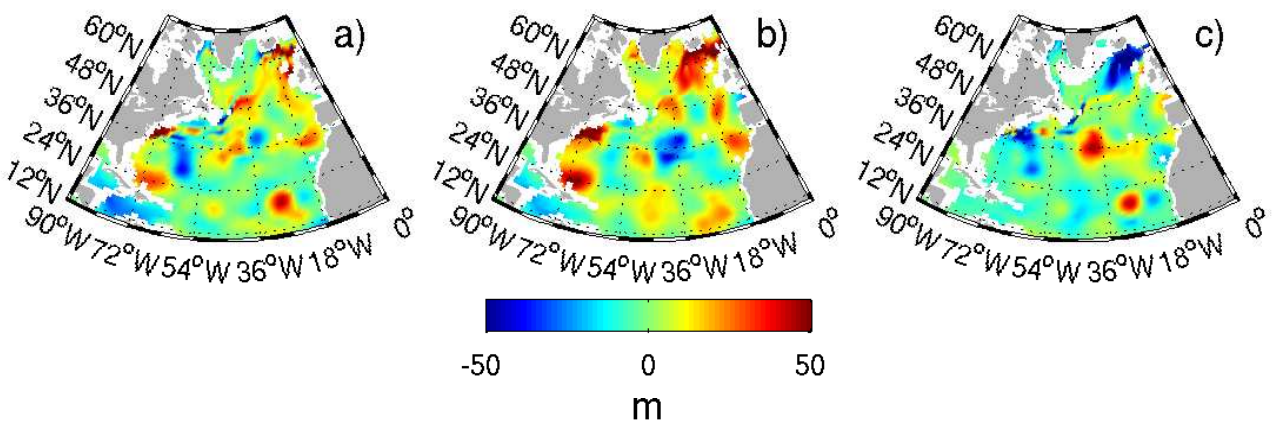

Fig. 5. The variation of the depth of the neutral surface of 27.5 between 2004 and 2003 due to both temperature and salinity anomalies (left), temperature only (middle) and salinity only (right).

similar to the full data set (Fig. 2, lower panel) with the correlation coefficient of 0.84 (the data were filtered with a 5 point low pass filter). The trend of the ASH decreases from $1.44 \mathrm{~mm} /$ year (error of $0.37 \mathrm{~mm} /$ year) to $0.84 \mathrm{~mm} /$ year (error of $0.42 \mathrm{~mm} /$ year) when data coverage is reduced from full to $50 \%$, respectively.

The choice of a reference depth $D$ is not a trivial problem. It should be deep enough so that the steric height is not sensitive to its further increase (this surface does not necessarily exist). Our experiments show that the depth of $1000 \mathrm{~m}$ is a good first approach for $D$ in the North Atlantic. We checked that computing ASH referenced to $1500 \mathrm{~m}$ does not change conclusions drawn here and opted for a shallower depth of $1000 \mathrm{~m}$ to have a better data coverage.

The total steric height anomaly $h_{a}$ can be split into two contributions, $h_{a}=h_{a 1}+h_{a 2}$, one from the water column between the surface and depth of seasonal thermocline $h_{s}\left(h_{a 1}\right)$ and the other between $h_{s}$ and $D\left(h_{a 2}\right)$. The total anomaly is dominated by $h_{a 1}$ over the seasonal cycle. The seasonal cycle in mid latitudes is much more profound in temperature than in salinity field. The changes of heat content in the seasonal thermocline are mainly a consequence of the net surface heat flux (Yan et al., 1995; Stammer, 1997). As a result changes of the steric height due to the seasonal thermocline can be calculated by using net surface heat flux. The deep part $h_{a 2}$ becomes a major contribution on longer (interannual) time scales or if the seasonal cycle of steric height is removed and salinity anomaly can be an important contributor to this part.

Usually the thermosteric and halosteric contributions to the steric height anticorrelate (see Fig. 4), i.e. partly compensate each other. This is not surprising, because the motion below the seasonal thermocline occurs along isopycnals and the salinity of a moving water parcel must be higher if its temperature is higher to retain the same density.

\section{Conclusions}

The analysis above shows that both temperature and salinity determine the variability of steric height in the North Atlantic over a 5-year period considered here. Considering only temperature variability leads to a basin averaged trend of $2.7 \mathrm{~mm} /$ year which is about of two-fold larger than the total trend in the steric height. Although this value is closer to the trend in basin averaged altimeter anomalies this closeness is only superficial. There is indeed improvement in the agreement between trends after inclusion of salinity anomalies as is best seen in zonal averages (see Fig. 4). On the local scale, the contribution from temperature and salinity are of similar magnitude.

The positive temporal trend in the surface height (from satellite altimetry) is higher than the trend in steric height only. It implies that other contributions of non-steric nature also play an important role in the North Atlantic over time scales studied here.

Acknowledgements. We are grateful to S. Esselborn for providing the altimeter data. VOI was supported by the UK NERC (RAPID program) whilst preparing the objective analysis of the Argo data.

Edited by: N. C. Wells

\section{References}

Antonov, J. I., Levitus, S., and Boyer, T. P.: Steric sea level variations during 1957-1994: Importance of salinity. J. Geophys. Res., 107(C12), 8013, doi:10.1029/2001JC000964, 2002.

Bretherton, F. P., Davis, R. E., and Fandry, C. B.: A technique for objective analysis and design of oceanographic experiments applied to MODE-73, Deep-Sea Res., 23, 559-582, 1976.

Gandin, L. S.: Objective analysis of meteorological fields. Leningrad, Gidrometeoizdat, 287 pp. (in Russian), English translation No. 1373 by Israel Program for Scientific Translations, (1965), 242 pp., 1963

Gill, A.E.: Atmosphere-Ocean Dynamics. Academic Press, 662 pp., 1982. 
Gilson, J., Roemmich, D., Cornuelle, B., and Fu, L.-L.: Relashionship of TOPEX/Poseidon altimetric height to steric height and circulation in the North Pacific, J. Geophys. Res., 103, 12, 27 947-27 965, 1998.

Ivchenko V. O., Wells, N. C., and Aleynik, D. L.: Anomaly of heat content in the Northern Atlantic in the last 7 years: is the ocean warming or cooling?, Geophys. Res. Lett., 33, L22606, doi:10.1029/2006GL027691, 2006.

Lavender, K. L., Owens, W. B., and Davis, R. E.: The mid-depth circulation of the subpolar North Atlantic Ocean as measured by subsurface floats, Deep-Sea Res. , 52, 767-785, 2005.

Maes, C.: Estimating the influence of salinity on sea level anomaly in the ocean, Geophys. Res. Lett., 25(19), 3551-3554, 1998.

Stammer, D.: Steric and wind-induced changes in TOPEX/POSEIDON large-scale sea surface topography observations, J. Geophys. Res., 102, 9, 20 987-21 009, 1997.

Stephens, C., Antonov, J. I., Boyer, T. P., Conkright, M. E., Locarnini, R., and O'Brien, T. D.: World Ocean Atlas 2001, 1: Temperature (CD-ROM), eds. by S. Levitus, NOAA Atlas NESDIS 49, U.S. Govt. Printing Office, Washington, D. C., 2002.
White, W. and Tai, C.-K.: Inferring interannual changes in global upper ocean heat storage from TOPEX altimetry, J. Geophys. Res., 100, 24 943-24 954, 1995.

Willis J. K., Roemmich, D., and Cornuelle, B.: Combining altimetric height with broadscale profile data to estimate steric height, heat storage, subsurface temperature, and seasurface temperature variability. J. Geophys. Res., 108, C93292, doi:10.1029/2002JC001755, 2003.

Willis J. K., Roemmich, D., and Cornuelle, B.: Interannual variability in upper ocean heat content, temperature, and thermosteric expansion on global scales, J. Geophys. Res., 109, C12036, doi:10.1029/2003JC002260, 2004.

Yan, X.-H., Niiler, P. P., Nadiga, S. K., Stewart, R. H., and Cayan, D. R.: Seasonal heat storage in the North Pacific: 1976-1989, J. Geophys. Res., 100, 6899-6926, 1995. 